\title{
Perioperative moxifloxacin treatment in rats subjected to deep hypothermic circulatory arrest: Reduction in cerebral inflammation but without improvement in cognitive performance
}

\author{
Kristine Kellermann, DVM, ${ }^{\mathrm{a}}$ Nadja Dertinger, DVM, ${ }^{\mathrm{a}}$ Manfred Blobner, MD, ${ }^{\mathrm{a}}$ Frieder Kees, DSc, \\ Eberhard F. Kochs, MD, ${ }^{a}$ and Bettina Jungwirth, $\mathrm{MD}^{\mathrm{a}}$
}

\begin{abstract}
Objective: Moxifloxacin reduces infectious complications after cerebral damage, such as ischemia and stroke. This study investigated whether moxifloxacin treatment influences cerebral inflammation and improves cognitive outcome after cardiopulmonary bypass with deep hypothermic circulatory arrest in rats.
\end{abstract}

\begin{abstract}
Methods: Rats were randomly assigned to deep hypothermic circulatory arrest $(n=40)$, sham operation $(n=40)$, and untreated control $(n=20)$ groups. Deep hypothermic circulatory arrest and sham groups were equally subdivided into moxifloxacin and placebo subgroups, receiving $6 \times 100 \mathrm{mg} / \mathrm{kg}$ moxifloxacin or saline solution every 2 hours intraperitoneally. Hippocampal tumor necrosis factor $\alpha$, nuclear factor $\kappa \mathrm{B}$, cyclooxygenase 2, and macrophages were assessed immunohistochemically. Histologic outcome was determined with hematoxylin and eosin. Neurologic outcome was assessed preoperatively and postoperatively. Cognitive performance was tested with the modified hole board test for 14 postoperative days.
\end{abstract}

Results: On postoperative day 14, deep hypothermic circulatory arrest moxifloxacin group was lower than deep hypothermic circulatory arrest placebo group in hippocampal neurons positive for tumor necrosis factor $\alpha(1.33$, $0.73-2.37$, vs $4.10,2.42-18.67)$, nuclear factor $\kappa \mathrm{B}(3.03,1.33-5.20$, vs $9.32,2.53-24.14)$, and cyclooxygenase 2 $(3.16,0.68-6.04$, vs 8.07, 3.27-19.91) and also had fewer macrophages than all other groups (72, 60-90, vs deep hypothermic circulatory arrest placebo 128, 76-203, sham moxifloxacin 89, 48-96, and sham placebo 81, 47-87). On postoperative day 14, both deep hypothermic circulatory arrest groups showed impaired motor, cognitive, and histologic outcomes relative to sham-operated groups, with no difference between deep hypothermic circulatory arrest subgroups.

Conclusions: Moxifloxacin transiently reduces cerebral inflammatory reaction, but without impact on neurologic function, histologic outcome, or long-term cognitive performance. (J Thorac Cardiovasc Surg 2011;141:796-802)

Anti-inflammatory substances such as the fluoroquinolone moxifloxacin (a Bayer product), a synthetic broad-spectrum antibiotic, can reduce infectious complications after such forms of cerebral damage as ischemia and stroke. This effect has been demonstrated after middle cerebral artery occlusion in mice, with moxifloxacin reducing poststroke infection and ameliorating neurologic outcome. ${ }^{1}$ This effect has been attributed mainly to the anti-infective activity of the substance, with infection presenting a severe consequence of stroke. ${ }^{2}$ In addition to its well-known anti-infective characteristics, moxifloxacin provides immunomodulatory properties. Although it has been less well described,

From Klinik für Anaesthesiologie, ${ }^{a}$ Klinikum rechts der Isar, Munich, Germany; and the Department of Pharmacology, ${ }^{b}$ University of Regensburg, Regensburg, Germany. Supported by Bayer Vital GmbH, Leverkusen, Germany.

Disclosures: Frieder Kees reports lecture fees and grant support from Bayer Health Care.

Received for publication Jan 26, 2010; revisions received March 19, 2010; accepted for publication April 16, 2010; available ahead of print June 21, 2010.

Address for reprints: Bettina Jungwirth, MD, Klinik für Anaesthesiologie, Technische

Universität München, Ismaninger Strasse 22, D-81675 Munich, Germany (E-mail:

b.jungwirth@1rz.tum.de).

0022-5223/\$36.00

Copyright (c) 2011 by The American Association for Thoracic Surgery

doi: $10.1016 /$ j.jtcvs. 2010.04 .017 immunomodulation is considered equally important for reducing inflammation and improving survival in vitro and in vivo. ${ }^{3,4}$ The significance of immunomodulation alone on functional outcome, without infection, is not yet known.

The rat model of cardiopulmonary bypass (CPB) with deep hypothermic circulatory arrest (DHCA) allows investigation of cerebral inflammation without clinical and biochemical signs of infection. ${ }^{5}$ The initiated inflammatory cascade includes the activation of tumor necrosis factor $\alpha(\mathrm{TNF} \alpha),{ }^{6}$ as well as nuclear transcription factor $\kappa \mathrm{B}$ $(\mathrm{NF} \kappa \mathrm{B}){ }^{7}$ This study was designed to investigate whether moxifloxacin treatment primarily influences the cerebral inflammatory reaction on a biochemical or histologic level and to determine whether moxifloxacin treatment secondarily improves cognitive or motor function after $\mathrm{CPB}$ with DHCA in rats.

\section{MATERIALS AND METHODS \\ Animals and Study Design}

After institutional animal care committee approval, 100 male SpragueDawley rats (330-400 g; Charles River, Sulzfeld, Germany) were housed under standard laboratory conditions (12 hours light/12 hours dark, $22^{\circ} \mathrm{C}$, $60 \%$ humidity, and free access to water and standard laboratory rat diet) for 3 weeks before the experiments for acclimatization. 

Abbreviations and Acronyms
COX-2 = cyclooxygenase 2
$\mathrm{CPB}=$ cardiopulmonary bypass
DHCA $=$ deep hypothermic circulatory arrest
$\mathrm{NF} \kappa \mathrm{B}=$ nuclear transcription factor $\kappa \mathrm{B}$
$\mathrm{TNF} \alpha=$ tumor necrosis factor $\alpha$

A total of 100 rats were randomly assigned to DHCA $(n=40)$, sham operation $(n=40)$, and control $(n=20)$ groups. These groups were then subdivided, with half of each respective group (DHCA $n=20$, sham $n=20$, control $\mathrm{n}=10$ ) allowed to survive for 24 hours. The remaining half of each group (DHCA $\mathrm{n}=20$, sham $\mathrm{n}=20$, control $\mathrm{n}=10$ ) was allowed to survive for 14 days. The DHCA and sham-operated groups were further split equally into moxifloxacin $(\mathrm{n}=10)$ and placebo $(\mathrm{n}=10)$ groups, receiving either moxifloxacin $(6 \times 100 \mathrm{mg} / \mathrm{kg}$ intraperitoneally every 2 hours, beginning with the onset of anesthesia) or a placebo (saline solution and $1 \mathrm{~mol} / \mathrm{L}$ hydrochloric acid, adjusted to $\mathrm{pH} 7$ with sodium hydroxide). The DHCA group rats were anesthetized, cannulated, connected to $\mathrm{CPB}$, and subjected to 45 minutes of DHCA. The sham group rats were anesthetized and surgically cannulated but not connected to $\mathrm{CPB}$, whereas rats in the control group remained untreated. Short-term cerebral inflammatory response and neurologic and histologic outcomes were studied in animals killed after 24 hours; long-term motor and cognitive function were studied in animals killed on the 14th postoperative day. Long-term histologic outcome on postoperative day 14 was also determined.

\section{CPB and DHCA}

Rats were intubated and mechanically ventilated with oxygen in air at an inspired oxygen fraction of $40 \%$ (arterial $\mathrm{PCO}_{2}, 32-40 \mathrm{~mm} \mathrm{Hg}$ ). All instruments were disinfected, catheters were gas sterilized, and surgery was carried out in an aseptic manner. Surgical sites were infiltrated with $2 \%$ lidocaine (Xylocaine). During surgical preparation, anesthesia was maintained with $2 \%$ to $2.5 \%$ isoflurane and $5-\mu \mathrm{g}$ fentanyl bolus doses. Heparin (150 IU) was administered. The tail artery (aortic inflow), the right superficial epigastric artery (blood pressure monitoring), and the right external jugular vein (venous drainage) were cannulated as previously described. ${ }^{5}$ Pericranial and rectal temperatures were monitored.

CPB was set up as previously described. ${ }^{5}$ The setup consisted of a venous reservoir, a peristaltic pump, a membrane oxygenator with integrated water quench, and an arterial inflow cannula, all connected by 1.6-mm internal diameter plastic tubing. An in-line flow probe (2N806 flow probe and T208 volume flowmeter; Transonics Systems, Inc, Ithaca, NY) was used to continuously measure CPB flow. CPB was instituted at a flow rate of 160 to 180 $\mathrm{mL} /(\mathrm{min} \cdot \mathrm{kg})$ and was consecutively decreased by half during the cooling period. With rectal temperature reaching $15^{\circ} \mathrm{C}$ to $18^{\circ} \mathrm{C}$ after 30 minutes, the roller pump was turned off and venous blood was drained to the reservoir. Circulatory arrest, as confirmed by asystole and lack of measurable mean arterial pressure, was maintained for $45 \mathrm{~min}$ at $15^{\circ} \mathrm{C}$ to $18^{\circ} \mathrm{C}$. With the reinstitution of $\mathrm{CPB}$, rewarming started at a rectal temperature of $15^{\circ} \mathrm{C}$ to $18^{\circ} \mathrm{C}$ and a flow rate of $105 \mathrm{~mL} /(\mathrm{min} \cdot \mathrm{kg})$. This inflow rate was gradually increased, reaching the full rate of 160 to $180 \mathrm{~mL} /(\mathrm{min} \cdot \mathrm{kg}$ ) at the end of the rewarming phase. Once a rectal temperature of at least $35.5^{\circ} \mathrm{C}$ was reached, $\mathrm{CPB}$ was terminated.

During $\mathrm{CPB}$, anesthesia consisted of $0.8 \%$ to $1 \%$ isoflurane, cisatracurium besylate (INN cisatracurium besilate, $1.6 \mathrm{mg} / \mathrm{h}$ ), and fentanyl bolus doses $(5 \mu \mathrm{g})$. A continuous positive airway pressure mode $\left(5 \mathrm{~cm} \mathrm{H}_{2} \mathrm{O}\right.$, inspired oxygen fraction 0.21) was applied to avoid atelectasis. During DHCA, anesthesia was discontinued. During rewarming, mean arterial pressure was kept above $50 \mathrm{~mm} \mathrm{Hg}$ by norepinephrine as soon as a rectal temperature of $30^{\circ} \mathrm{C}$ and a blood flow of $150 \mathrm{~mL} /(\mathrm{min} \cdot \mathrm{kg})$ were achieved. Arterial blood gas values were controlled with the $\mathrm{pH}$-stat strategy (arterial $\mathrm{PCO}_{2}$ of 31 to $40 \mathrm{~mm} \mathrm{Hg}$ ).

After cessation of $\mathrm{CPB}$, rats remained anesthetized with $1.0 \%$ to $1.5 \%$ isoflurane, intubated, and ventilated for 1 hour (rectal temperature $36.5^{\circ} \mathrm{C}$ ). Bicarbonate was administered to treat acidosis, and calcium was injected to prevent a drop of ionized calcium below $1 \mathrm{mmol} / \mathrm{L}$. The heparin-induced anticoagulation was allowed to dissipate spontaneously. When animals resumed spontaneous breathing, the tracheas were extubated and the rats were placed under continuous observation for 12 hours in a transparent, oxygen-enriched box with free access to water and food. Animals were returned to their cages at the first postoperative day and were housed in their familiar groups. According to group assignment, rats were killed either at 24 hours or 14 days after CPB with DHCA. Animals were anesthetized, serum samples were harvested, and brains were removed and snap frozen on dry ice and stored at $-80^{\circ} \mathrm{C}$ for further analyses.

\section{Assessment of Biochemical Outcomes}

The concentrations of moxifloxacin were determined in serum samples harvested 24 hours after DHCA and in brain tissue homogenates with a reversephase high-performance liquid chromatographic method with fluorimetric detection at an excitation frequency of $396 \mathrm{~nm}$ and an emission frequency of 504 $\mathrm{nm} .{ }^{8}$ The limits of quantification (signal to noise ratio 10/1) for serum brain tissue moxifloxacin were $20 \mathrm{ng} / \mathrm{mL}$ and $20 \mathrm{ng} / \mathrm{g}$, respectively.

Serum samples of all groups taken 24 hours after DHCA were analyzed for TNF $\alpha$ levels by enzyme-linked immunosorbent assay with a minimum detectable level of $5 \mathrm{pg} / \mathrm{mL}$ (Qantikine; R\&D Systems, WiesbadenNordenstadt, Germany).

To detect hippocampal neurons positive for $\mathrm{TNF} \alpha$ (polyclonal rabbit anti-TNF $\alpha$; Acris Antibodies, Hiddenhausen, Germany), NF $\kappa$ B (polyclonal rabbit anti-NF $\kappa \mathrm{B}$ p65; Abcam, Cambridge, UK), and cyclooxygenase 2 (COX-2; monoclonal rabbit anti-COX-2, Cell Signaling; Beverley, Mass), an immunohistochemical double staining was performed (7 $\mu \mathrm{m}$; $3.3 \mathrm{~mm}$ caudal from bregma [bregma $-3.3 \mathrm{~mm}$ ]) for each inflammatory marker. Neurons were labeled with an anti-neuronal nuclei antibody (Mouse Anti-Neuronal Nuclei Monoclonal Antibody; Millipore, Billerica, Mass). Neurons positive for $\mathrm{TNF} \alpha, \mathrm{NF} \kappa \mathrm{B}$, and COX-2 were counted throughout the hippocampus under a light microscope $(\times 400)$.

Macrophages of rats killed at 24 postoperative hours were marked (CD68 mouse anti-human macrophage marker; United States Biological Inc, Swampscott, Mass) on brain slices (7 $\mu \mathrm{m}$; bregma $-3.3 \mathrm{~mm}$ ) and counted throughout the hippocampus $(\times 400)$.

\section{Assessment of Histologic Outcome}

Cell damage in the hippocampus was assessed in brain slices $(7 \mu \mathrm{m}$; bregma $-3.3 \mathrm{~mm}$ ) stained with hematoxylin and eosin (according to the number of eosinophilic neurons throughout the hippocampus $(\times 400)$ of the rats killed at 24 postoperative hours. To represent the complexity of ischemic damage 14 days after DHCA, a modified score was used. ${ }^{9}$ The number of intact neurons was graded on an incremental 3-point scale $(0$ representing $100 \%$ intact neurons, 1 representing $99 \%-95 \%$ intact neurons, 2 representing $94 \%-84 \%$ intact neurons, and 3 representing $\leq 83 \%$ intact neurons). In addition, numbers of cerebral infarcts were assessed and scored on an incremental 3-point scale (for size: 0 representing no infarction, 1 representing 1 to $5 \mathrm{~mm}^{2}, 2$ representing 6 to $10 \mathrm{~mm}^{2}$, and 3 representing more than $10 \mathrm{~mm}^{2}$; for number: 0 representing no infarcts, 1 representing 1 infarct, 2 representing 2 infarcts, and 3 representing more than 2 infarcts). Score values were summed to provide a total histologic score for each animal, with the score varying between 0 (normal brain) and 12 (maximal damage).

\section{Assessment of Functional Outcomes}

To assess short-term motor function, animals underwent standardized functional neurologic testing both on the preoperative day and on the day 

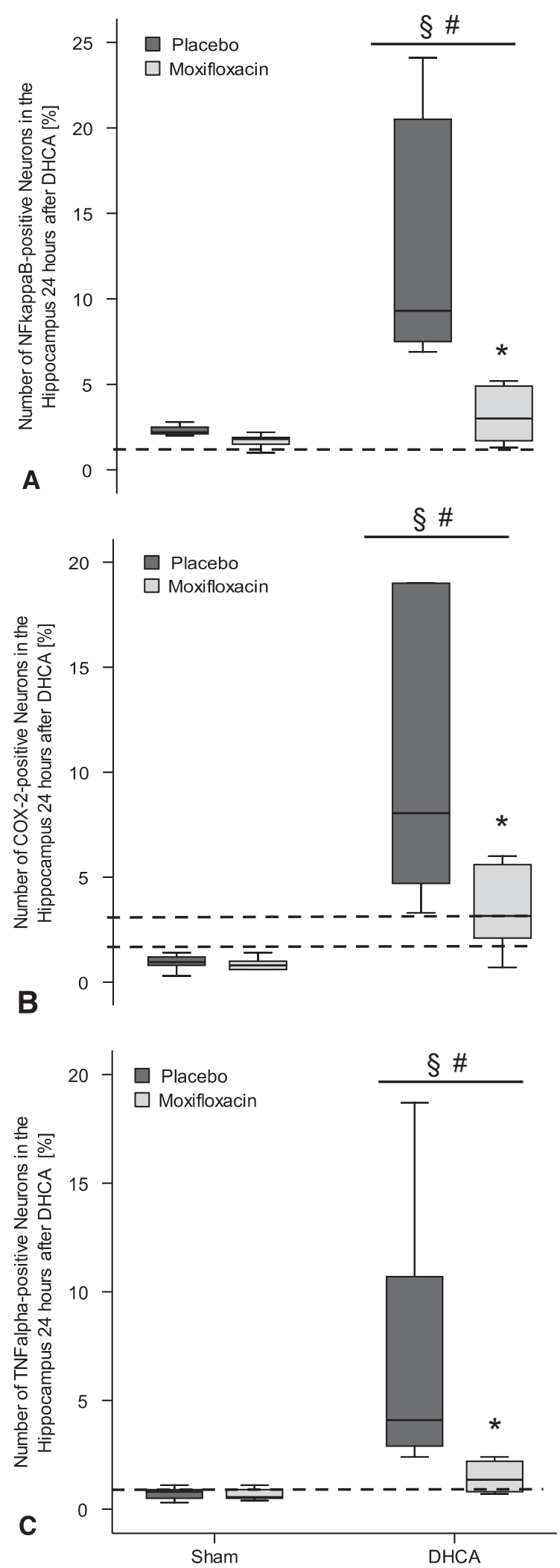

FIGURE 1. Inflammatory parameters nuclear factor $\kappa \mathrm{B}$ (NFkappaB), cyclooxygenase $2(C O X-2)$, and tumor necrosis factor $\alpha$ (TNFalpha) in hippocampus 1 day after deep hypothermic circulatory arrest $(D H C A)$. A, Deep they were killed. Motor function was scored from performances on a beam and a rotating grid, time hanging on a rope, movement symmetry, and gait. ${ }^{5}$ Consequently, each animal tested received score values from 0 through 18 , with 0 to 1 representing normal motor function.

For the assessment of long-term outcome, animals were allowed to survive for 14 postoperative days. At the end of the experiment, motor function was assessed as described previously, complemented by 2 fine motor skill parameters from the modified hole board test (time per food intake, number of abortive hole visits). Cognitive and behavioral function were assessed with daily testing in the modified hole board test according to an established protocol. ${ }^{10}$ Briefly, the rats lived in an area divided into home cage $(80 \times 60 \times 50 \mathrm{~cm})$ and test arena $(40 \times 60 \times 50 \mathrm{~cm})$, with the hole board $(20 \times 40 \mathrm{~cm})$ placed in the middle of the test arena. Fifteen cylinders were staggered on the board and baited with almonds underneath a grid. Three cylinders that were marked with white tape contained almonds above the grid and, therefore, obtainable for the rats. All cylinders were treated with black currant aroma to cover smell of the almonds.

On the second and third postoperative days, animals were habituated to the hole board and the almonds. Beginning with the fourth postoperative day, rats learned the test until postoperative day 14. Animals were tested in the modified hole board test for 3 trials daily. The sequence of marked cylinders was randomly changed every day.

Motor and cognitive function were scored as previously described,,${ }^{5}$ with every animal receiving score values from 0 to 24 for motor and cognitive performance and 0 to 22 for behavior, with 0 to 1 representing normal motor, cognitive, and behavioral function.

\section{Statistics and Data Management}

Data are presented as box and whisker plots. Biochemical, histologic, and functional outcomes were analyzed with the Kruskal-Wallis test with post hoc Mann-Whitney U. Motor function and cognitive performance were assessed with a previously described scoring system. ${ }^{5}$ Individual functional parameters were recorded as score values (maximum scores being the worst outcome) and summed to total motor scores (0-18 and 0-24, respectively) and a total cognition score $(0-24)$ for each animal at the end of the experiment. Statistical analyzes were performed with SPSS version 16.0 for Windows (SPSS Inc, an IBM Company, Chicago, Ill).

\section{RESULTS}

Nine animals were excluded from further data analysis because of cannulation problems $(n=3)$ or insufficient venous

hypothermic circulatory arrest placebo group shows highest numbers of hippocampal neurons positive for nuclear factor $\kappa \mathrm{B}$ relative to all other groups. Data are presented as box and whisker plot. Asterisk indicates $P<.001$ versus deep hypothermic circulatory arrest placebo; section mark indicates $P<.001$ versus sham moxifloxacin; crosshatch indicates $P<.001$ versus sham placebo. Dashed line marks 90th percentile of untreated controls. B, Placebo group shows highest numbers of hippocampal neurons positive for cyclooxygenase 2 relative to sham groups and untreated controls. Data are presented as box and whisker plot. Asterisk indicates $P=.007$ versus deep hypothermic circulatory arrest placebo; section mark indicates $P<.001$ versus sham moxifloxacin; crosshatch indicates $P<.001$ versus sham placebo. Dashed lines mark 10th and 90th percentiles of untreated controls. C, Deep hypothermic circulatory arrest placebo group shows highest numbers of hippocampal neurons positive for tumor necrosis factor $\alpha$ relative to all other groups. Data are presented as box and whisker plot. Asterisk indicates $P<.001$ versus deep hypothermic circulatory arrest placebo; section mark indicates $P<.001$ versus sham moxifloxacin; crosshatch indicates $P<.001$ versus sham placebo. Dashed line marks 90th percentile of untreated controls. 
return $(n=6)$. These rats were replaced to keep sample size equal.

Moxifloxacin was detectable in brain and serum at 24 postoperative hours, with significantly higher levels in the DHCA group than in the sham group (brain DHCA group, $1.52 \pm 0.24$ and sham group, $0.28 \pm 0.07 \mu \mathrm{g} / \mathrm{g}$; serum DHCA group, $5.71 \pm 0.93$ and sham group, $1.25 \pm 0.03$ ). Serum TNF $\alpha$ levels at 24 hours after DHCA, assessed with enzyme-linked immunosorbent assay, were below the limit of quantification of $5 \mathrm{pg} / \mathrm{mL}$ (DHCA groups, $1.16 \pm$ $0.26 \mathrm{pg} / \mathrm{mL}$; sham groups, $0.98 \pm 0.22 \mathrm{pg} / \mathrm{mL}$ ). In the early postoperative phase, hippocampal $\mathrm{TNF} \alpha, \mathrm{NF} \kappa \mathrm{B}$, and $\mathrm{COX}-$ 2-expressions were significantly higher in the DHCA placebo group than in the DHCA moxifloxacin group. The higher expressions of inflammatory parameters in the DHCA placebo group were accompanied by elevated numbers of macrophages in the hippocampus relative to all other groups (Figures 1 and 2).

At both 24 hours and 14 days after CPB with DHCA, histologic outcomes were impaired in both DHCA groups relative to the sham groups. There was no effect of moxifloxacin treatment (Figure 3).

At 24 postoperative hours, motor function was impaired in both DHCA groups relative to sham groups, with no effect of moxifloxacin treatment (Figure 4). At 14 postoperative days, both motor and cognitive functions were impaired in both DHCA groups relative to sham groups, with no effect of moxifloxacin treatment. Behavioral parameters were comparable among all experimental groups (Figure 5).

\section{DISCUSSION}

Moxifloxacin transiently reduced inflammation in the brain 24 hours after CPB with DHCA in rats, but without any influence on short-term histologic and neurologic outcomes. This early anti-inflammatory effect of moxifloxacin did not alter motor and cognitive performance 14 days after CPB with DHCA.

The recovery model of DHCA in rats induces both motor and neurocognitive deficits, dependent on the duration of DHCA. In this study, 45 minutes of DHCA was chosen to induce a minor cerebral insult with a high survival rate but sufficiently detectable effects on both neurologic and neurocognitive performances. ${ }^{5}$ As intended, rats showed postoperative impairments in cognitive function and motor deficits after DHCA relative to sham-operated rats. The histologic examination correspondingly revealed neuronal cell damage. Even more important for studying the impact of an anti-inflammatory drug, ${ }^{11}$ however, is that this model of DHCA induced a severe inflammatory reaction in placebotreated rats, as evidenced by higher numbers of macrophages and upregulation of neuronal $\mathrm{TNF} \alpha, \mathrm{NF} \kappa \mathrm{B}$, and COX-2 levels.

We found clinically relevant amounts of moxifloxacin ${ }^{12}$ in both the brain and the serum 24 hours after DHCA,

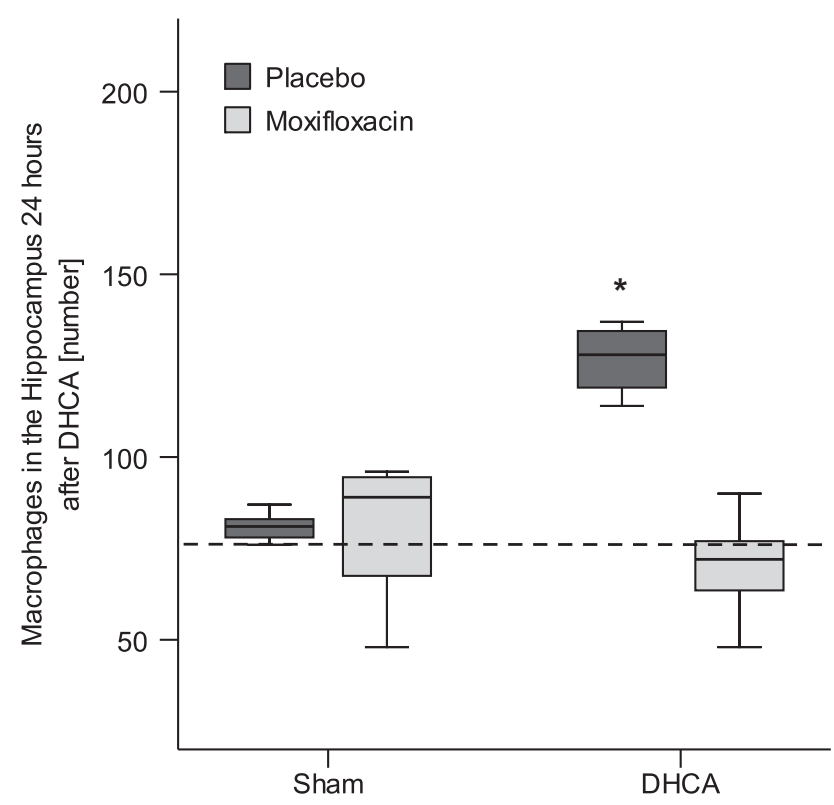

FIGURE 2. Macrophages in hippocampus 1 day after deep hypothermic circulatory arrest (DHCA). Number of macrophages in hippocampus was elevated in deep hypothermic circulatory arrest placebo group relative to all other groups. Data are presented as box and whisker plot. Asterisk indicates $P=.001$ versus all other groups. Dashed line marks 90th percentile of untreated controls.

whereas the sham-operated moxifloxacin-treated rats showed only minor concentrations. In both groups, though, moxifloxacin was equally distributed between serum and brain tissue. The 4-fold higher moxifloxacin concentration in the DHCA group 20 hours after last administration of the drug was most likely due to slower metabolism and reduced tissue perfusion in this group, leading to the prolongation of the substance's half-life.

Cerebral moxifloxacin significantly reduced the number of macrophages in this early postoperative phase and simultaneously curbed neuronal expressions of $\mathrm{TNF} \alpha$, $\mathrm{NF} \kappa \mathrm{B}$, and $\mathrm{COX}-2$ in the hippocampus at 24 postoperative hours. The effect on macrophages and their secretory products $^{13}$ is of particular interest, because fluoroquinolones are suspected to inhibit the activation of early cell signaling events associated with the inflammatory response ${ }^{14}$ with moxifloxacin for example directly inhibiting the interleukin $1 \beta$-induced nuclear translocation of $\mathrm{NF} \kappa \mathrm{B}^{4}$

The previously mentioned early anti-inflammatory effect of moxifloxacin in the hippocampus, however, had no benefit in terms of histologic damage. At both 24 hours and 14 days after DHCA, histologic outcome was unaffected by the moxifloxacin treatment, with both the placebo and the moxifloxacin-treated groups showing equal increases in eosinophilic neurons and tissue damage. In addition, no particular effect of moxifloxacin treatment on the functional 


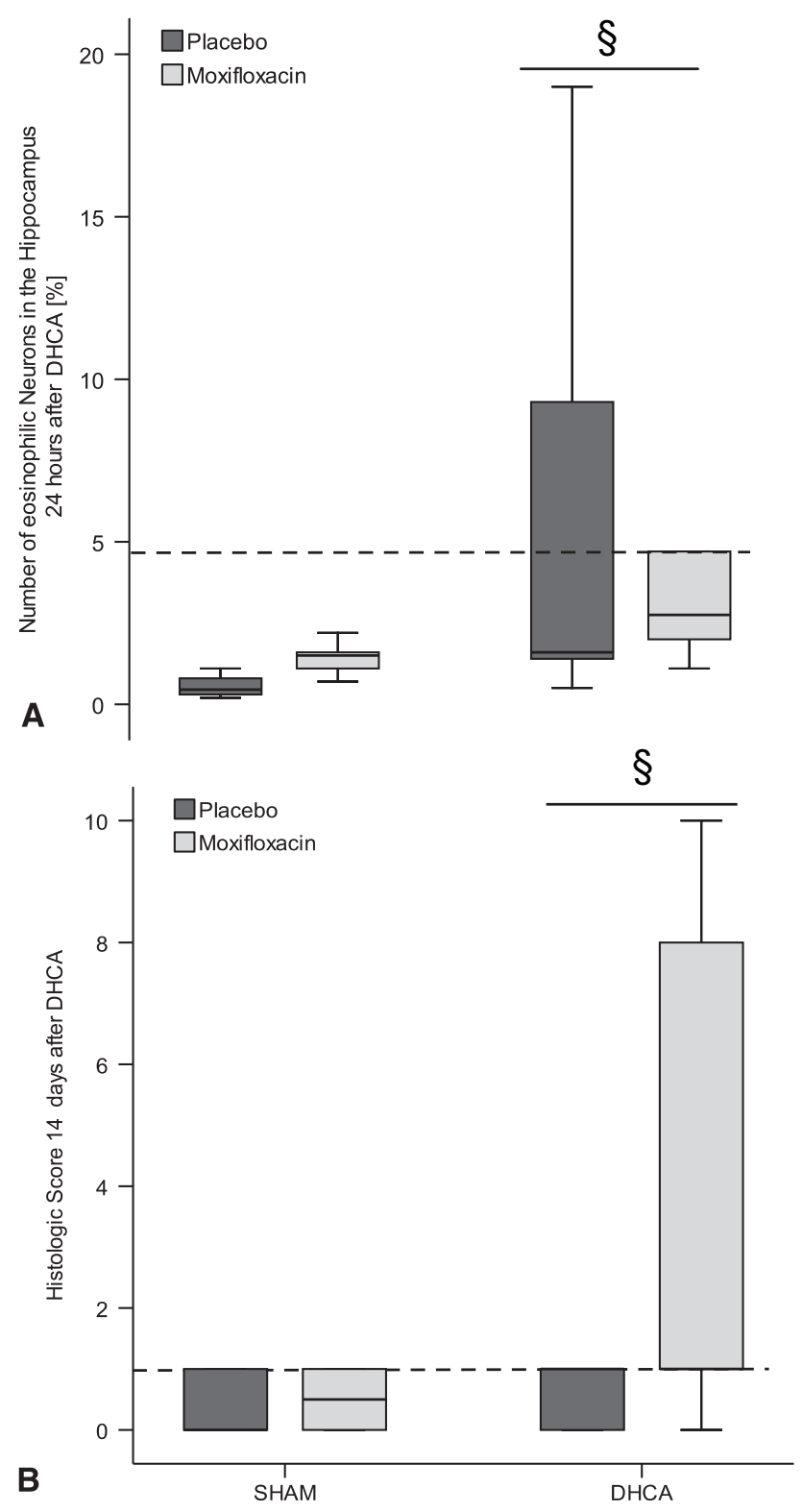

FIGURE 3. Histologic outcomes 1 and 14 days after deep hypothermic circulatory arrest (DHCA). A, At 1 day after deep hypothermic circulatory arrest, both deep hypothermic circulatory arrest groups show increased numbers of eosinophilic neurons in hippocampus. Data are presented as box and whisker plot. Section mark indicates $P<.001$ versus sham. Dashed line marks 90th percentile of untreated control animals. B, At 14 days after deep hypothermic circulatory arrest, both deep hypothermic circulatory arrest groups show worse histologic outcomes. Data are presented as box and whisker plot. Section mark indicates $P=.006$ versus sham. Dashed line marks 90 th percentile of untreated control animals.

outcome was detected. Looking at the early postoperative period, moxifloxacin treatment did not improve motor function 24 hours after DHCA. The long-term neurocognitive performance remained equally unaffected by moxifloxacin, despite a marked downregulation of inflammatory parame-

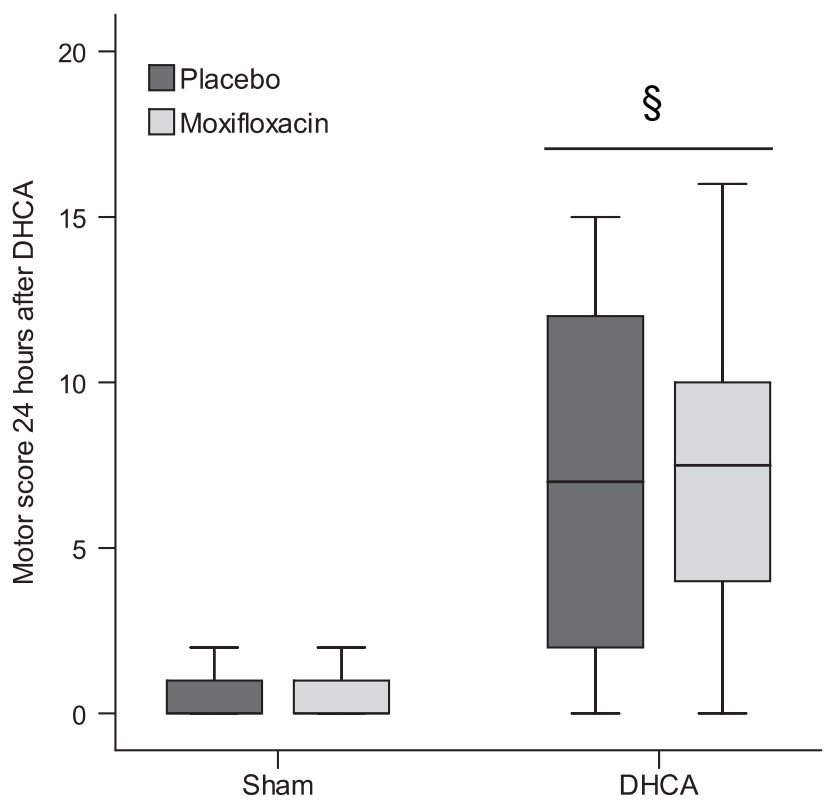

FIGURE 4. Motor function 1 day after deep hypothermic circulatory arrest (DHCA) was equally impaired in both deep hypothermic circulatory arrest groups relative to preoperative baseline and to sham groups. Preoperative baseline is not depicted ( 0 in all 4 groups). Data are presented as box and whisker plot. Section mark indicates $P<.001$ versus sham.

ters in the early postoperative phase in the hippocampus, a formation known to be crucial for learning and memory function. ${ }^{15}$

Considering our results, one wonders why moxifloxacin treated-mice have shown a better outcome after stroke. ${ }^{1}$ The answer is likely to be found within the essential difference between stroke and CPB with DHCA. Stroke is followed by secondary infection, such as pneumonia, which accounts for most associated morbidity and mortality. ${ }^{2}$ CPB with DHCA in our model, in contrast, triggers an inflammatory response without infection. ${ }^{6,7}$ In consequence, one would expect the antibiotic moxifloxacin to improve outcome after infection in the setting of a stroke model. ${ }^{1}$

Moxifloxacin reduced cerebral levels of $\mathrm{TNF} \alpha, \mathrm{NF} \kappa \mathrm{B}$, and COX-2 24 hours after CPB with DHCA in this study but had no impact on histologic and functional outcomes. This finding adds to the current debate about significance of brain inflammation after bypass surgery in terms of cognitive outcome, recently questioned in an editorial by Nussmeier and Searles. ${ }^{16}$ Reviewing and discussing the most recent findings, Nussmeier and Searles ${ }^{16}$ concluded that the inflammatory response to CPB is likely not the primary driver of neurocognitive injury after CPB. This notion has been confirmed by the results of this study.

Finally, some important limitations remain. This study was performed in young, healthy animals without any comorbidities identified as risk factors for adverse cerebral outcome, such as diabetes, congenital heart disease, or 

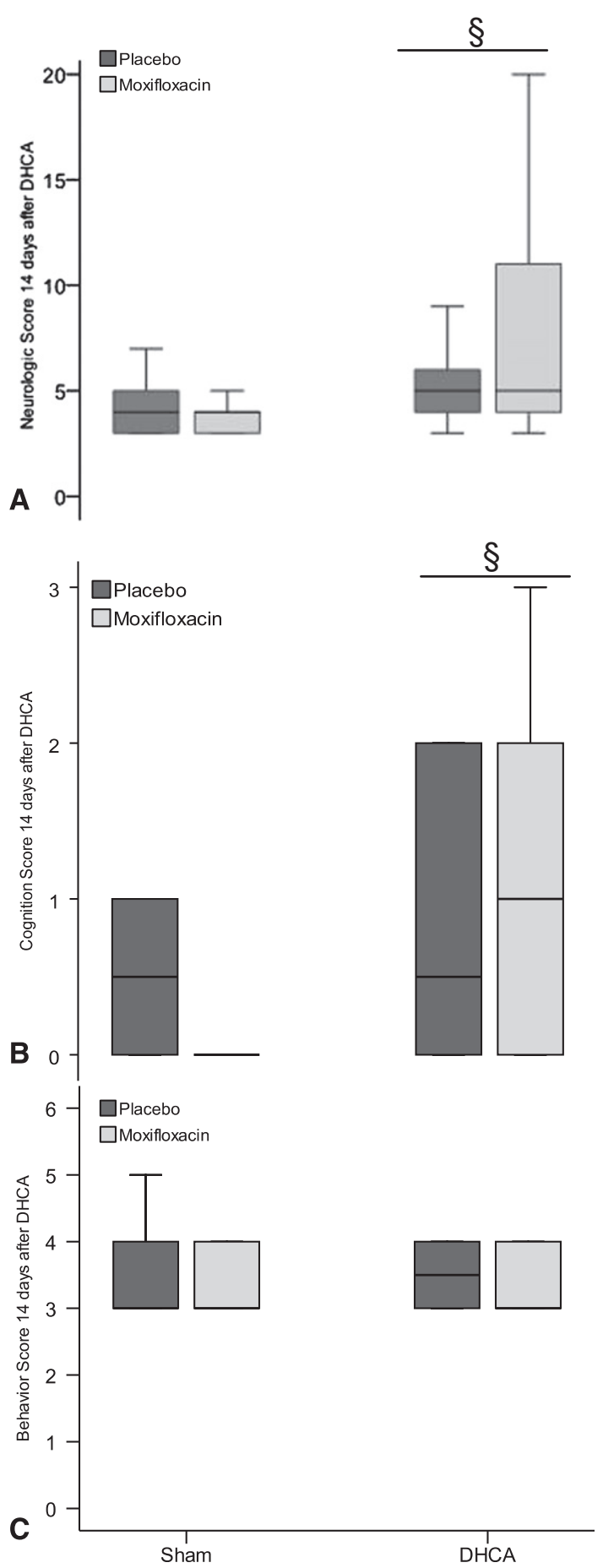

FIGURE 5. Motor function, cognitive performance, and behavior 14 days after deep hypothermic circulatory arrest (DHCA). A, Long-term motor function was similarly affected in both deep hypothermic circulatory arrest groups Section mark indicates $P=.018$ versus sham. B, Both deep hypothermic circulatory arrest groups showed equally impaired cognitive performance 14 days after deep hypothermic circulatory arrest. Section mark indicates $P=.021$ versus sham. C, Behavior showed no difference among all 4 groups. Data are presented as box and whisker plots. Score (value 0 ) for untreated control is not depicted. significant atheromatous disease of the aorta, such as may be found in the aging patient population presenting for cardiac surgery. ${ }^{17}$ In addition, because only a single dose of moxifloxacin with a particular treatment regimen $(6 \times 400 \mathrm{mg} /$ $\mathrm{kg}$ distributed through 10 hours) was investigated, we cannot rule out the possibility that a different dosage or treatment scheme might have an impact on functional outcome in this particular setting.

\section{CONCLUSIONS}

Moxifloxacin penetrates the blood-brain barrier during CPB with DHCA in rats. In the brain, it transiently ameliorates the cerebral inflammatory reaction in the absence of postoperative infection. The fact that this significantly reduced cerebral inflammation had no impact on histologic or biochemical outcome adds another piece of evidence toward the theory that inflammation after CPB is likely an epiphenomenon and not the main culprit in postoperative neurocognitive dysfunction.

We thank Simone Denzler for neurologic and neurocognitive testing of the animals and Johannes Stückle for performing laboratory analyses.

\section{References}

1. Meisel C, Prass K, Braun J, Victorov I, Wolf T, Megow D, et al. Preventive antibacterial treatment improves the general medical and neurological outcome in a mouse model of stroke. Stroke. 2004;35:2-6.

2. Emsley HC, Hopkins SJ. Acute ischaemic stroke and infection: recent and emerging concepts. Lancet Neurol. 2008;7:341-53.

3. Shalit I, Horev-Azaria L, Fabian I, Blau H, Kariv N, Shechtman I, et al. Immunomodulatory and protective effects of moxifloxacin against Candida albicansinduced bronchopneumonia in mice injected with cyclophosphamide. Antimicrob Agents Chemother. 2002;46:2442-9.

4. Werber S, Shalit I, Fabian I, Steuer G, Weiss T, Blau H. Moxifloxacin inhibits cytokine-induced MAP kinase and NF- $\kappa \mathrm{B}$ activation as well as nitric oxide synthesis in a human respiratory epithelial cell line. J Antimicrob Chemother. 2005; 55:293-300.

5. Jungwirth B, Mackensen GB, Blobner M, Neff F, Reichart B, Kochs EF, et al. Neurologic outcome after cardiopulmonary bypass with deep hypothermic circulatory arrest in rats: description of a new model. J Thorac Cardiovasc Surg. 2006; 131:805-12.

6. Jungwirth B, Kellermann K, Qing M, Mackensen GB, Blobner M, Kochs EF. Cerebral tumor necrosis factor alpha expression and long-term neurocognitive performance after cardiopulmonary bypass in rats. $J$ Thorac Cardiovasc Surg. 2009; 138:1002-7.

7. Jungwirth B, Eckel B, Blobner M, Kellermann K, Kochs EF, Mackensen GB. The impact of cardiopulmonary bypass on systemic interleukin- 6 release, cerebral nuclear factor-kappa B expression, and neurocognitive outcome in rats. Anesth Analg. 2010;110:312-20.

8. Beckmann J, Kees F, Schaumburger J, Kalteis T, Lehn N, Grifka J, et al. Tissue concentrations of vancomycin and Moxifloxacin in periprosthetic infection in rats. Acta Orthop. 2007;78:766-73.

9. Barber PA, Hoyte L, Colbourne F, Buchan AM. Temperature-regulated model of focal ischemia in the mouse: a study with histopathological and behavioral outcomes. Stroke. 2004;35:1720-5.

10. Ohl F, Holsboer F, Landgraf R. The modified hole board as a differential screen for behavior in rodents. Behav Res Methods Instrum Comput. 2001;33: 392-7.

11. Pulsinelli WA, Brierley JB, Plum F. Temporal profile of neuronal damage in model of transient forebrain ischemia. Ann Neurol. 1982;11:491-8.

12. Grayo S, Lott-Desroches MC, Dussurget O, Respaud R, Fontanet A, JoinLambert $\mathrm{O}$, et al. Rapid eradication of Listeria monocytogenes by moxifloxacin 
in a murine model of central nervous system listeriosis. Antimicrob Agents Chemother. 2008;52:3210-5.

13. Nathan CF. Secretory products of macrophages. J Clin Invest. 1987;79:319-26.

14. Purswani M, Eckert S, Arora H, Johann-Liang R, Noel GJ. The effect of three broad-spectrum antimicrobials on mononuclear cell responses to encapsulated bacteria: evidence for down-regulation of cytokine mRNA transcription by trovafloxacin. J Antimicrob Chemother. 2000;46:921-9.
15. Eichenbaum H. Hippocampus: cognitive processes and neural representations that underlie declarative memory. Neuron. 2004;44:109-20.

16. Nussmeier NA, Searles BE. Inflammatory brain injury after cardiopulmonary bypass: is it real? Anesth Analg. 2010;110:288-90.

17. Karimi A, Ahmadi H, Davoodi S, Movahedi N, Marzban M, Abbasi K, et al. Factors affecting postoperative morbidity and mortality in isolated coronary artery bypass graft surgery. Surg Today. 2008;38:890-8.

Access to The Journal of Thoracic and Cardiovascular Surgery Online is reserved for print subscribers!

Full-text access to The Journal of Thoracic and Cardiovascular Surgery Online is available for all print subscribers. To activate your individual online subscription, please visit The Journal of Thoracic and Cardiovascular Surgery Online, point your browser to http://www.mosby.com/jtcvs, follow the prompts to activate your online access, and follow the instructions. To activate your account, you will need your subscriber account number, which you can find on your mailing label (note: the number of digits in your subscriber account number varies from 6 to 10). See the example below in which the subscriber account number has been circled:

\section{Sample mailing label}

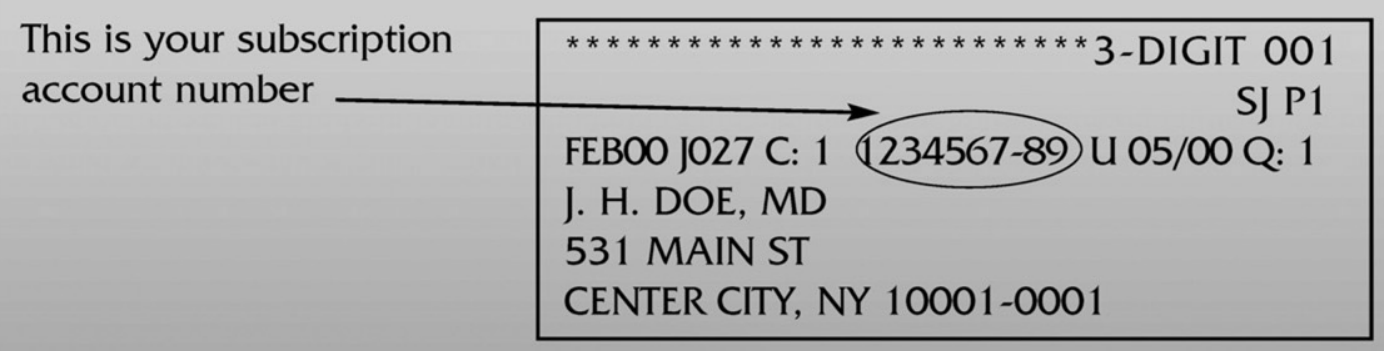

Personal subscriptions to The Journal of Thoracic and Cardiovascular Surgery Online are for individual use only and may not be transferred. Use of The Journal of Thoracic and Cardiovascular Surgery Online is subject to agreement to the terms and conditions as indicated online. 